\title{
Does interferometry work? A critical look at the foundations of interferometric surface topography measurement
}

Peter de Groot, Xavier Colonna de Lega, Rong Su, Richard Leach

Peter de Groot, Xavier Colonna de Lega, Rong Su, Richard Leach, "Does interferometry work? A critical look at the foundations of interferometric surface topography measurement," Proc. SPIE 11102, Applied Optical Metrology III, 111020G (3 September 2019); doi: 10.1117/12.2526654

Event: SPIE Optical Engineering + Applications, 2019, San Diego, California, United States 


\title{
Does interferometry work? A critical look at the foundations of interferometric surface topography measurement
}

\author{
Peter de Groot ${ }^{1}$, Xavier Colonna de Lega ${ }^{1}$, Rong $\mathrm{Su}^{2}$ and Richard Leach ${ }^{2}$ \\ ${ }^{1}$ Zygo Corporation, Laurel Brook Road, Middlefield, CT 06455 \\ ${ }^{2}$ University of Nottingham, Nottingham NG8 1BB, UK
}

\begin{abstract}
Interferometers for the measurement of surface form and texture have a reputation for high performance. However, the results for many types of surface features can deviate from the expectation of one cycle of phase shift per half wavelength of surface height. Here we review the fundamentals of imaging interferometry and describe ways of defining instrument response, including the linear instrument transfer function. These considerations define practical regimes of linear behavior that are usually satisfied for traditional uses of interferometers; but that are increasingly challenged by applications involving complex textures and high surface slopes. We conclude by proposing pathways for further improving performance on difficult surface structures using advanced modeling techniques.
\end{abstract}

Keywords: Interferometry, diffraction, interference, instrument transfer function, optical transfer function, metrology

\section{INTRODUCTION}

Interferometers are widely used in science and industry for the measurement of surface form and texture. Laser Fizeau interferometers measure up to meter-class optics [1], while interference microscopes reveal final surface details on lateral scales below a micrometer [2]. Commercial instruments achieve measurement noise levels over millions of data points below $0.1 \mathrm{~nm} / \sqrt{\mathrm{Hz}}$ [3]. Instruments are available that simultaneously measure flatness, thickness variation and material homogeneity for optical components [4], while other systems based on coherence scanning interferometry (CSI) provide process control for automotive parts [5] and semiconductor devices [6]. Modeling methods have added the ability to analyze surface materials and transparent film structures in addition to surface form and texture [7]. Methods have been developed to acquire data in difficult environments with air turbulence and vibration $[8,9]$. These advances have been motivated by the continued success of interferometry across an increasing range of applications.
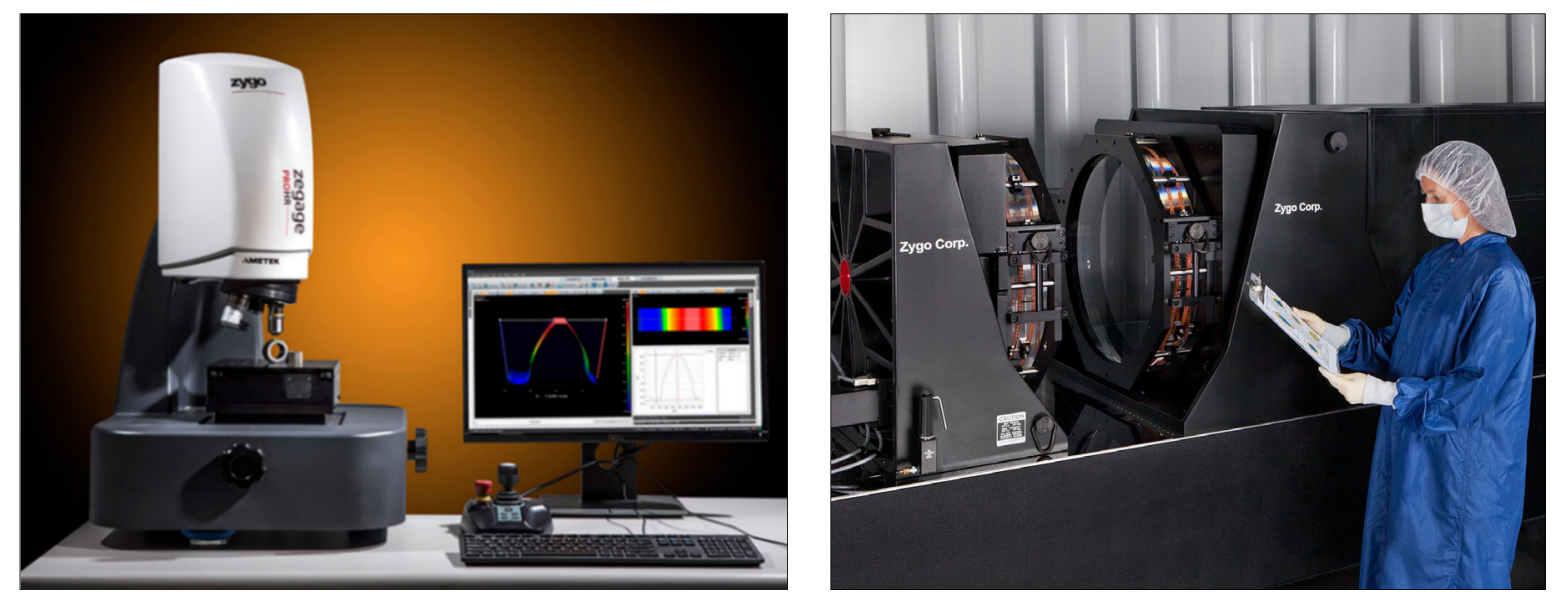

Figure 1: (Left) An interference microscope. (Right) A large-aperture laser Fizeau interferometer. 
Given the success of instruments such as those shown in Figure 1, it is safe to say that interferometry does work. Nonetheless, there are frequent occasions when interferometers do not work as expected. This is increasingly the case as traditional metrology techniques are applied to non-traditional test objects, from additive manufacturing parts to aspherical or freeform lenses with highly sloped surfaces [10-13]. The desire to extend the technique to these challenging new measurement tasks motivates a critical re-examination of the foundational principles of the technique.

\section{FUNDAMENTALS}

The foundational premise of interferometry for dimensional metrology is that interference fringe phase is proportional to an optical path difference. If for example we have a Michelson-type interferometer and the measurement mirror moves a distance $h$, we expect a phase shift inversely proportional to the wavelength $\lambda$

$$
\phi=\frac{4 \pi}{\lambda} h
$$

If we know the wavelength and its associated uncertainty, simple inversion of this equation provides a traceable measurement of length. It is natural to suppose that we can extend this measurement principle to areal surface topography measurements using an equation

$$
h(x, y)=\frac{\lambda}{4 \pi} \phi(x, y)
$$

where $x, y$ are coordinates in a plane that coincides with the surface, and $h$ is the local surface height along the orthogonal $z$ direction. A digital camera detects the interference signal over multiple surface points in the $x, y$ plane, and the phase follows from some form of spatial or temporal heterodyning to extract information from interference fringes. This idea is fundamental to the operating principle of interferometers.

Notwithstanding the attractiveness of a direct linear relationship between interference phase and surface height, it is understood that this is not quite correct for surface topography measurements. If for example two neighboring surface points are closer together than the imaging lateral resolution of the system, then we will not be able to distinguish between the corresponding surface heights at all. This tells us something critically important about interferometric instruments for surface topography measurements: They are, first and foremost, imaging systems. Given that interferometers for surface topography measurement rely on optical imaging, we might expect that the response of an interferometer to surface features would parallel that of a conventional imaging system. As we shall see, this is sometimes approximately the case; but it is by no means true in general.

\section{IMAGING OF 3D STRUCTURES}

First it is important to distinguish between reflectivity objects, which we will define as perfectly flat and having only a reflectivity pattern, and topography objects, which have a height variation $h(x, y)$. We then define a simple model of an optical system that includes an illuminator, the object surface, imaging optics, an image detector, and a data acquisition and processing method for generating quantitative data about the surface from the image. For further simplicity, the model assumes that the illumination is a monochromatic plane wave, thus defining a coherent optical system.

In both imaging and topography measurement, there is interest in the instrument response to individual sinusoidal components of the surface. For conventional imaging of reflectivity objects, a co-sinusoidal amplitude reflectivity pattern of unit contrast along the $x$ direction results in a light field $U_{R}$ that varies at the spatial frequency $v$ as follows:

$$
U_{R}(x, y)=1+\cos (2 \pi v x) .
$$


The Fourier transform of this wavefront has only three Fourier frequency components at $f=-v, 0, v$. From the classical grating equation, these frequencies correspond to the zeroth and first diffraction orders at angles $\alpha$ with respect to the $z$ axis given by

$$
\sin (\alpha)=\lambda f
$$

For a sinusoidal topography object of amplitude $b$, the reflected field $U_{h}$ is modulated in phase according to

$$
U_{h}(x, y)=\exp \left[\frac{4 \pi i}{\lambda} b \cos (2 \pi v x)\right]
$$

which expands to

$$
U_{h}(x, y)=a_{0}+i a_{1} \cos (2 \pi v x)+\sum_{p=2}^{\infty} i^{p} a_{p} \cos (p 2 \pi v x),
$$

where the coefficients are

$$
a_{p}=J_{p}\left(\frac{4 \pi b}{\lambda}\right)
$$

The first two terms of the light field $U_{h}$ for a topography object look similar to the field $U_{R}$ for a reflectivity object. However, these first two terms in $U_{h}$ are followed by a sum of contributions at higher spatial frequencies $p u$ that are not actually present in the surface structure $[14,15]$. Figure 2 illustrates this difference. As the amplitude $b$ increases, the number of diffracted beams also increases. One way to conceptualize the higher diffracted angles is to think of them as reflections from the more steeply-angled surfaces of the sinusoidal topography, similar to what takes place with blazed diffraction gratings.
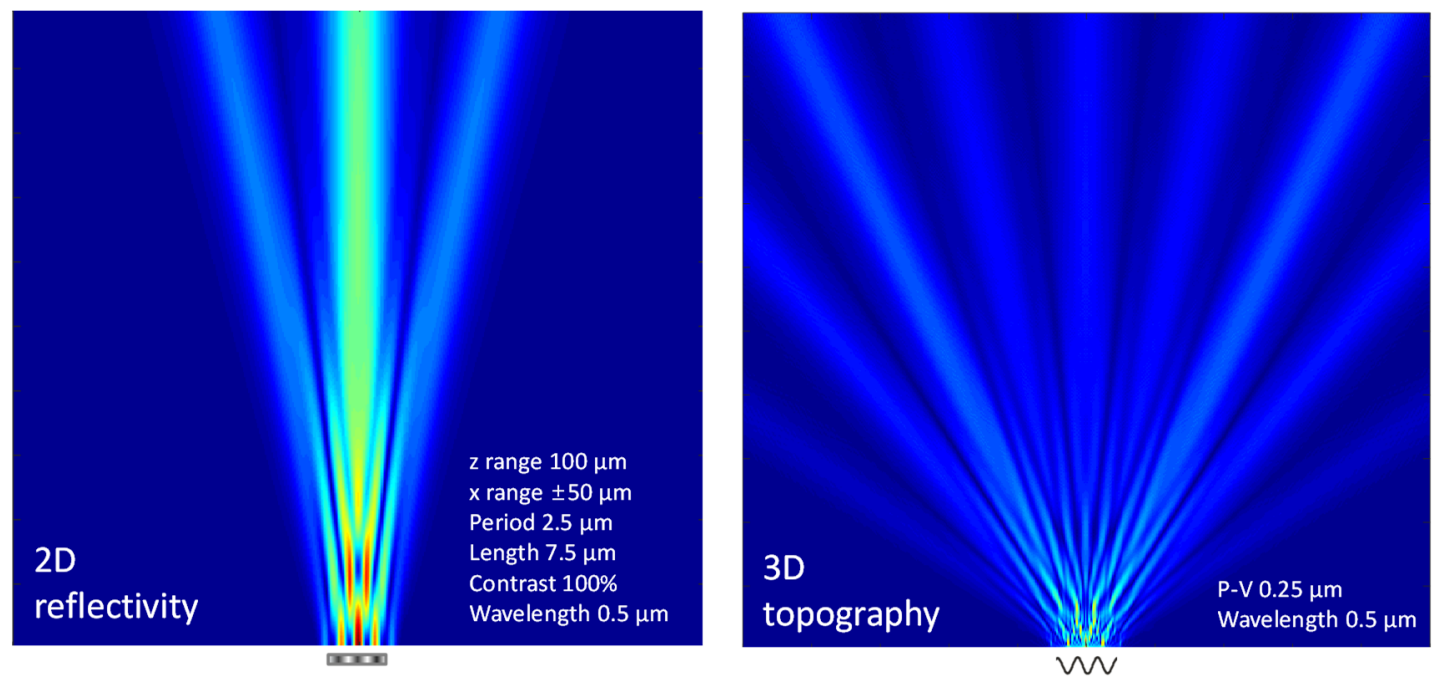

Figure 2: Illustration of the diffraction from a one-dimensional, sinusoidal reflectivity pattern (left) and a sinusoidal surface topography variation (right),with the same sinusoidal period and sample length for both object types 
There are some significant consequences to the differences in the way that light diffracts from these two types of object surfaces. In a coherent system, the scattered light will pass if the diffraction angles are no larger than those admitted by the entrance pupil of the imaging system, as defined by the numerical aperture

$$
A_{N}=\sin (\psi),
$$

where $\psi$ is the maximum acceptance angle for the entrance pupil in object space. This filtering effect on the propagating light field is linear in complex amplitude for all diffraction orders that are within the instrument's bandwidth [14]. It is clear from Figure 2 that for a given optical system and limiting aperture, it is possible that a reflectivity pattern is correctly imaged; whereas for a topography object the loss of higher-order diffactions could lead to errors. Even though the optical filtering effect is linear in this model, the instrument response using interference phase as a measure of surface topography may be nonlinear.

These observations regarding diffraction from 3D objects lead to the following requirement, which if satisfied, should allow us to reconstruct the topography correctly:

1. The optical system should have a sufficiently large numerical aperture to capture the light scattered from the topography object, including the higher diffraction orders.

This first condition is readily satisfied in the optical testing of the low-order surface form of polished flats and spheres, for example, in a laser Fizeau interferometer. A second criterion applies to sharp edges and step-like features, which scatter a continuum of diffraction angles that will always be blocked to some degree. In such cases, we can still have linear behavior if the topography is shallow enough that the range of diffraction angles is similar to that of a reflectivity object:

2. For discrete steps and steep walls, linear behavior is still possible if the size of the step is much less than one quarter of the mean wavelength.

If one or the other of these two "rules of the road" are satisfied, and there are no other sources of nonlinearity, then the instrument response will be at least approximately linear [16-18].

\section{THE INSTRUMENT TRANSFER FUNCTION}

Assuming that we are operating the instrument in the linear regime, the next step is to establish a means to quantify the instrument response using tools that parallel familiar methods from conventional optical imaging systems. Imaging instruments for reflectivity objects are often characterized by the modulation transfer function (MTF), which documents the image contrast as a function of spatial frequency for an incoherent optical system [19, 20]. Typically, the MTF declines with spatial frequency, eventually reaching a limit according to the resolving power or lateral resolution.

There is some ambiguity in the use of the MTF in practice. According to the ISO 9334 standard, the MTF is the magnitude of the optical transfer function (OTF), which itself is the Fourier transform of the incoherent point spread function (PSF) [19]. It is clear that the PSF relates only to the optics in an instrument - that is, the glass parts, limiting apertures, illumination geometry and so forth. However, in other contexts, the MTF can refer to a complete electro-optical assembly, including camera, electronics and data processing [20]. This is sometimes referred to as the system MTF, and in practice, it is this quantity that serves as a specification for instrument performance [21].

The parallel specification to the system MTF for interferometers is the instrument transfer function (ITF), which quantifies the height response as a function of the spatial frequency of the surface topography [16, 22]. One definition for the ITF $T(v)$ is the ratio of the power spectral density (PSD) of the topography at each surface spatial frequency $v$ :

$$
T(v)=\sqrt{P^{\prime}(v) / P(v)}
$$


where $P$ is the PSD, given by the square magnitude of the Fourier components of the known surface structure, and $P^{\prime}$ is the corresponding measured value [17, 23-25]. More generally, it can be defined as the ratio of Fourier components for the measured and true topography; in which case $T(v)$ may include a complex phase. In all cases, the ITF corresponds to an evaluation of the complete system, not just the optics. Indeed, it is possible to determine the ITF of an instrument without having any idea how it functions, by evaluating standard specimens [26-28]. The ITF is agnostic to the measurement technology, which could be based on contact stylus methods, ultrasonic testing, pneumatic gaging, or optical interferometry. As such, we can use the OTF or similar transfer function for the optics as part of a model used for predicting the ITF of an interferometer, but the OTF and the ITF are most definitely not the same thing.

Fundamental to the concept of an ITF is the fact that the instrument response is linear in spatial frequency within defined limits [22]. To be meaningful, the ITF must be independent of surface shape and shift invariant, at least locally within the field of view. It is improper to use ITF as a synonym for instrument response in general. It is possible, however, for an instrument to be nonlinear, part dependent and field dependent, and still to be useful; it just would not have a defined ITF. Similarly, a material measure for calibration that is inconsistent with the rules of the road defined above, is not an ITF specimen; but can be used nonetheless to evaluate instrument response for the specific surface structure of the material measure.

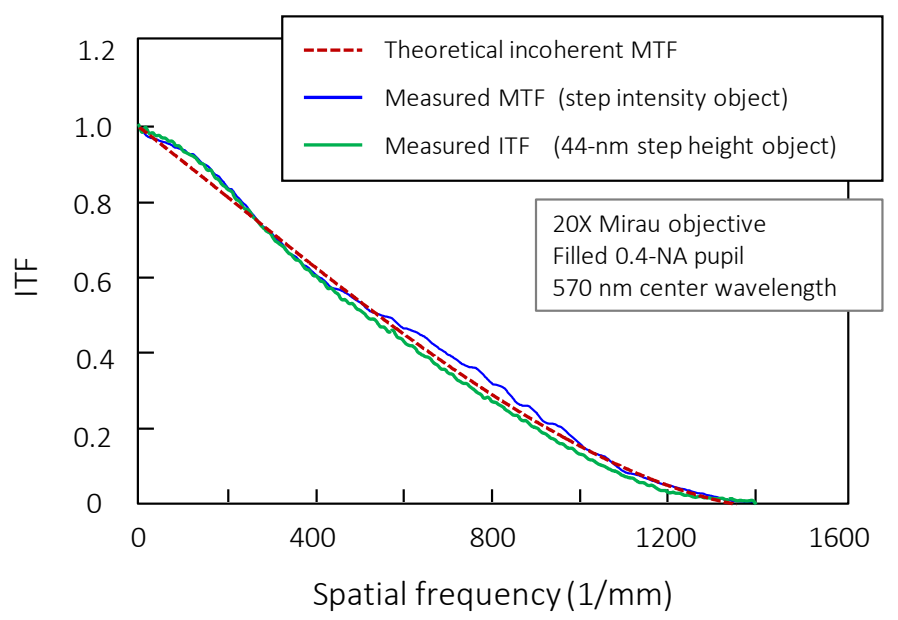

Figure 3: Comparison of the experimentally-determined ITF, the calculated optical imaging MTF, and the measured MTF for an interference microscope. A white-light LED provides the incoherent, broadband illumination.

The good news is that for a wide variety of instrument types and practical applications, the ITF follows the system MTF, and we can use this as a guide when designing an electro-optical system for interferometry [16]. Figure 3 illustrates an example of approximation of the ITF using the system MTF for an interference microscope. These data compare an experimental measurement of the ITF using a $44 \mathrm{~nm}$ step specimen with an experimental determination of the incoherent MTF using an opaque screen to obtain the line-spread function for the system [17].

The encouraging results of Figure 3 illustrate the expected performance of interferometers - that is, that they should behave like conventional imaging systems, with the addition of surface height information. However, even within the boundaries defined by the two rules stated above for slopes and discrete feature heights, it is still possible to have important differences with respect to imaging reflectivity objects, some of which relate to the fact that interferometers rely on just the phase of the imaged light field.

An important example of the difference between intensity and topography imaging within the linear regime is the laser Fizeau interferometer, which is a coherent optical system. The optical transfer function for coherent illumination, also known as the amplitude transfer function or ATF, preserves the magnitude of the spatial frequencies up to the cutoff defined by the limiting aperture, even in the presence of aberrations and focus errors [14]. In an interferometer, however, a focus error degrades the spatial frequency response, resulting in blurring, even in coherent light [29]. This is because the phase of the scattered light contributions captured by the instrument are affected by aberrations, even if the Fourier 
component magnitudes are not. In this case, the ITF does not necessarily follow the magnitude of the ATF, and a deeper analysis is essential. This effect has been thoroughly studied and is an important consideration in achieving optimal performance in modern interferometer design [30-32].

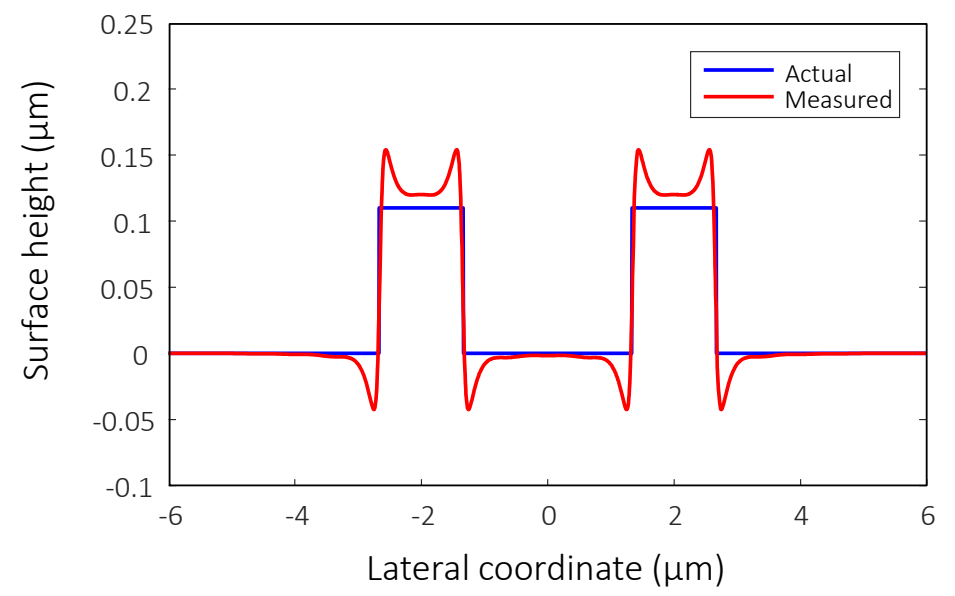

Figure 4: Simulation of a measurement of a square profile using synthetic wavelength interference microscopy. The measurement is for a feature height equal to $0.11 \mu \mathrm{m}$, using an NA $=0.4$, incoherent illumination at two wavelengths, $0.5 \mu \mathrm{m}$ and $0.6 \mu \mathrm{m}$.

Another important consideration is that many types of interferometers for surface topography measurement use multiple wavelengths or white light as a means to overcome the fringe-order ambiguity of monochromatic or laser-based methods. Examples include synthetic wavelength interferometry [30], which uses two or more discrete wavelengths, and coherence scanning interferometry [2], which may employ wavelengths covering a range of $100 \mathrm{~nm}$ in the visible region. These methods take advantage of the rate of change of interference phase with wavelength to measure a wide variety of objects, including high steps and rough textures. A known difficulty with these techniques is that the transfer characteristics of the optics are wavelength dependent [33,34]. Differences in the spatial frequency response with wavelength can result in large errors in the measured topography, particularly with steep slopes and sharp step-like features. Figure 4 illustrates this phenomenon for a two-wavelength interferometer using a simulation based on Fourier optics.
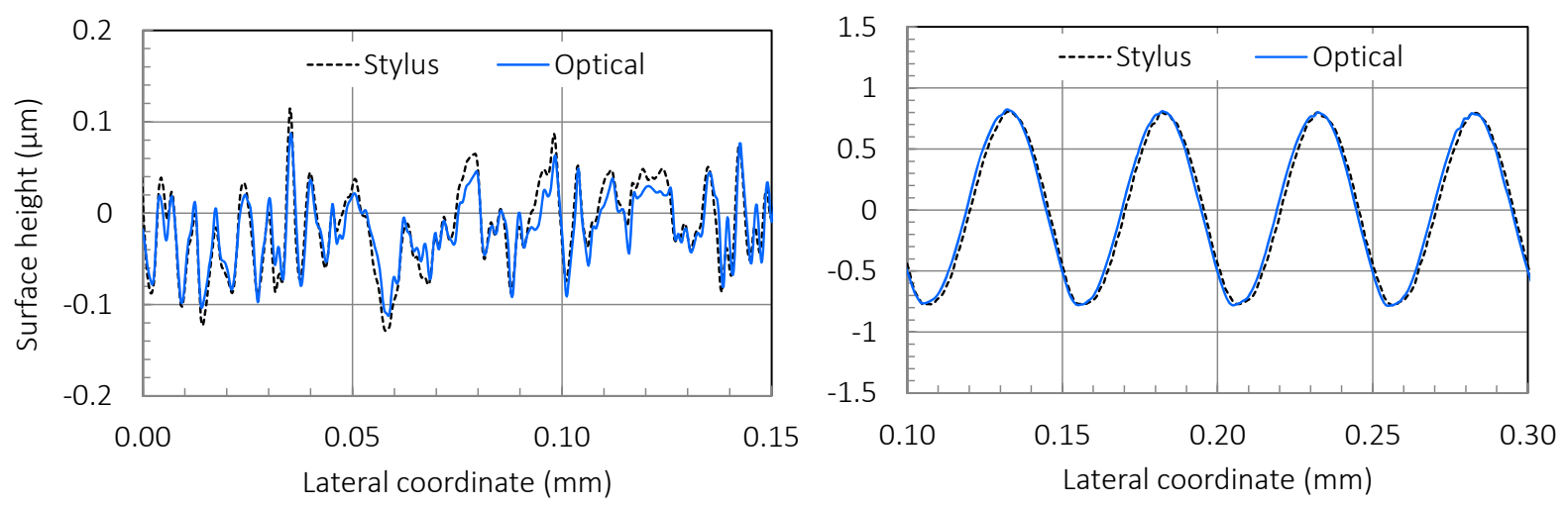

Figure 5: Comparison of profile measurements using a CSI microscope and a mechanical stylus instrument, illustrating good correlation between the two measurement techniques for random (left) and sinusoidal (right) samples.

The measurement distortions shown in Figure 4 are well known [35-37] and a number of practical methods for minimizing errors have been developed [38-40]. Figure 5 shows examples of a comparison of stylus profile measurements with optical interferometry results on roughness specimens. The measurements were performed at separate laboratories on the same samples, taking care to match the spatial frequency range for both instrument types, as detailed in Ref.[41]. These results 
illustrate linear behavior for both instruments within the slope range and spatial frequency content of the specimens, and correlation consistent with routine measurements of surfaces of this type. However, it is a practical reality that results are not always this pleasing, particularly when the optical instrument is challenged by higher slopes and step features.

\section{TAKING THE NEXT STEP-ADVANCED 3D OPTICAL MODELING}

Interferometry for surface topography is an active area of research, both in the development of new instruments and in extending the range of application for established platforms. Modeling methods are of special interest for understanding and quantifying errors, and for developing strategies for improved topographical fidelity and metrological traceability.

One approach to modeling optical systems involves diffraction calculations followed by numerical integration over an area of pupil positions, illumination directions and optical paths for each point in the field of view [42-44]. These models often rely on now-classical Fourier optics methods, involving sectioning light fields into a sequence of $x, y$ planes propagating in the orthogonal $z$ direction. Although this is a capable approach; it is worthwhile taking a step back and considering how best to tackle the problem of imaging $3 \mathrm{D}$ structures from a fresh perspective.

In conventional imaging of reflectivity objects, we often model the transfer characteristics using the PSF, which is the best-focused spot of light that a perfect lens with an aperture can make, limited by the diffraction of light $[14,19]$. In a circular-aperture, diffraction-limited optical system, the PSF is the familiar Airy pattern in the $x, y$ plane shown in Figure 6 (a). The PSF can be extended to $x, y, z$ space by mathematical propagation through a sequence of focus positions. If the light source has a broad spectral bandwidth, the focus is performed for each wavelength, and these contributions are added together by incoherent superposition. The result is the 3D PSF shown in cross section in Figure 6 (b). If we now model the complete interferometric system with a reference light field, we obtain the 3D interferometer impulse response shown in Figure 6 (c), which includes a modulation that can be equated to the interference signal that we would see if we moved the object with respect to the instrument through a range of $z$ positions.

The next step in the thought process is to consider this 3D interferometer impulse response as a kind of non-contact stylus probe [45]. The concept and the corresponding modeling methods are particularly well adapted to CSI, which involves scanning a measurement volume in the $z$ direction and using both the peak magnitude of the impulse response together with the underlying fringe structure to determine a local surface height value. Figure 7 (a) illustrates how this works for selected points on a sinusoidal topography, and Figure 7 (b) shows the result of convolving the interferometer impulse response with an imaginary thin surface layer or foil that represents the top of the surface in 3D [45]. In this image we can already see some important effects, such as the signal loss at high slopes.
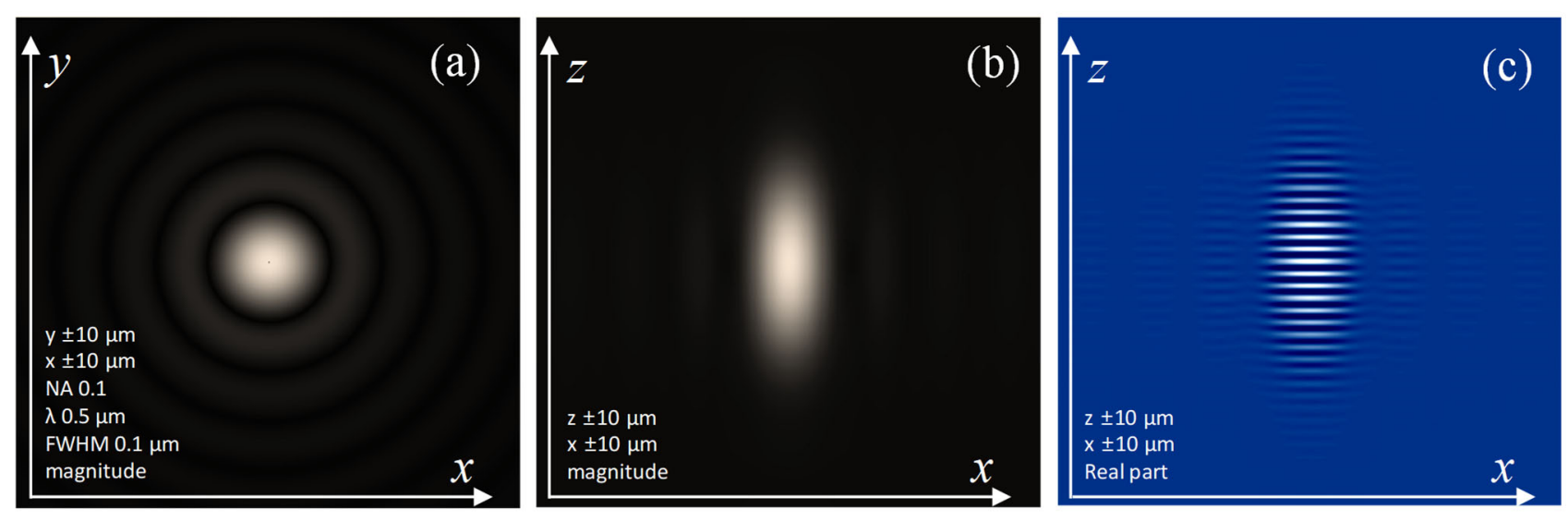

Figure 6: (a) Magnitude of the 2D PSF in the $x, y$ plane. (b) An $x, z$ cross section of the magnitude of the 3D PSF, constructed by propagating the 2D PSF through a range of focus positions. (c) The 3D interferometer impulse response. 

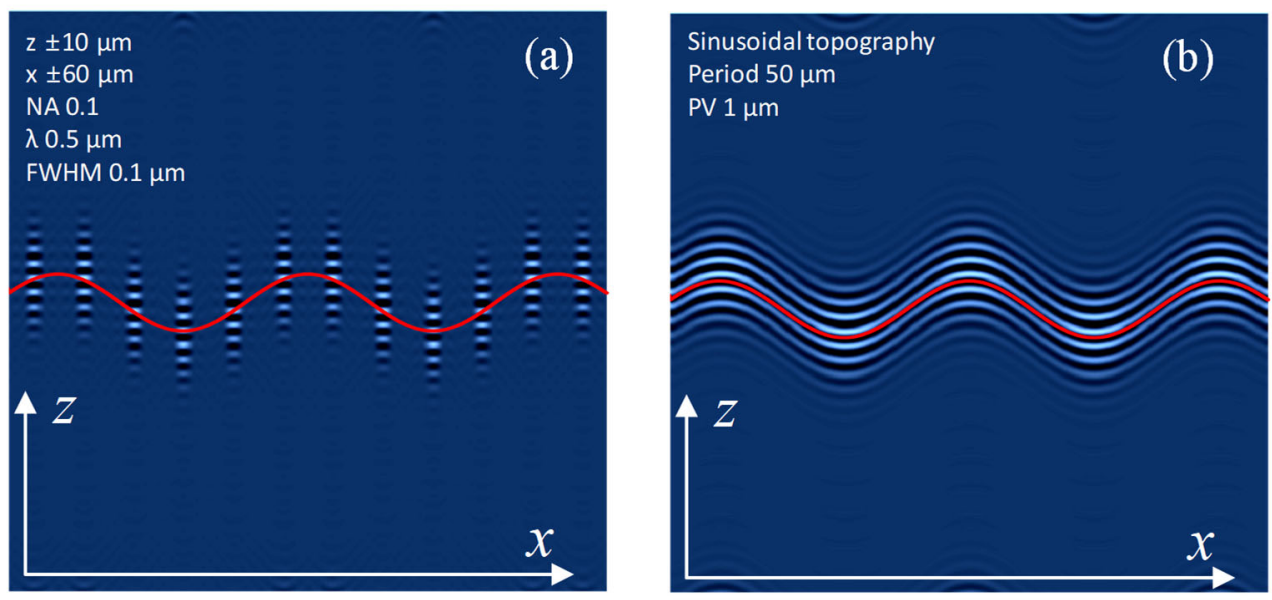

Figure 7 (a) Sample points on a sinusoidal topography showing the impulse response at each position. (b) Result of convolving the impulse response with the surface. The red line in both images represents the profile of the surface.

In addition to the qualitative appeal of the 3D impulse response for interferometers, there are quantitative advantages, particularly at higher NA values for which the impulse response is shaped as much by focus as by spectral bandwidth [46]. The 3D Fourier transform of the impulse response provides a 3D transfer function that fully describes the filtering process from the foil-like surface to the imaged light field [47, 48]. The analogous 3D OTF is well established in advanced modeling of optical imaging and metrology systems, particularly in holography and for confocal microscopy, and can be derived from first principles in electromagnetic theory [49-51].

An important application of the 3D transfer function approach is for the calibration of optical aberrations, focus effects, and wavelength-dependent response. The calibration can be performed with a sphere of known radius and high quality, so as to present a full range of known scattering angles and phase offsets to the instrument [52]. After calibration, inverse filtering of the transfer function can correct for many of the errors encountered in interferometry. Figure 8 illustrates this capability for a smooth, sinusoidal topography variation and a CSI microscope with a $50 \times$ Mirau objective.

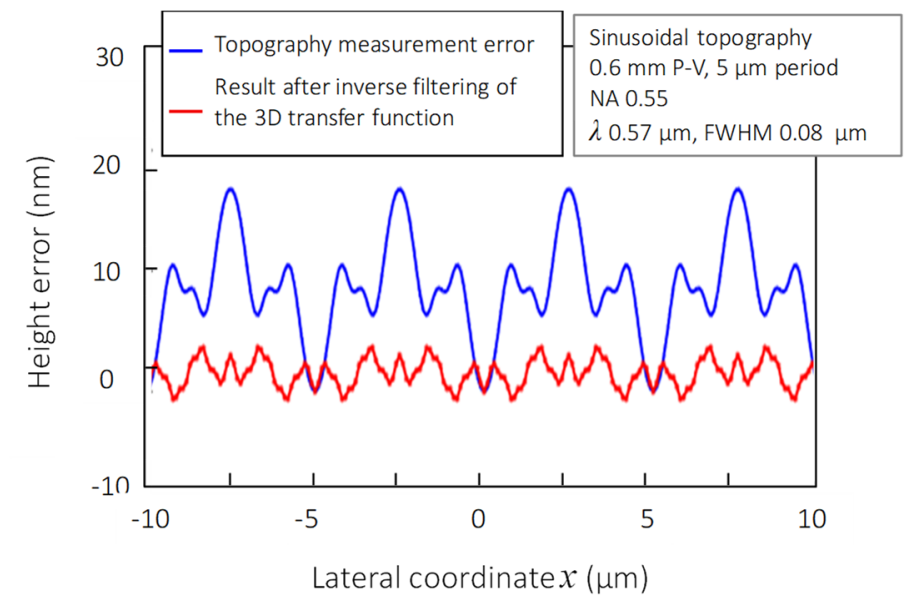

Figure 8: Comparison of a topography measurement error on a roughness specimen using interference microscopy before (blue) and after (red) correction using 3D impulse response modeling. 


\section{SUMMARY}

It is natural and common to think of measuring surface topography by assuming that the fringe phase is directly and linearly proportional to surface height in an interferometer. However, as we have seen, it is not quite as simple as that. First, we must take into account that scattering from a topography surface is more complicated than it is for a reflectivity object, usually involving higher diffraction orders. Properly imaging the object light field requires that we either capture all of these diffraction orders, or that we limit ourselves to surface variations that are small when compared to the wavelength. Additional considerations relate to the fundamental differences between detecting and reporting the intensity of the imaged light field, and extracting surface topography information using only the complex phase of the light.

Fortunately, for the most traditional applications of interferometric surface topography measurement, the complexities of interferometric 3D imaging can often be reduced to familiar concepts of linear transfer functions. If for example the instrument response is linear and can be characterized by Fourier analysis of the reported surface topography, then we can define an ITF that is analogous to (but not the same as) the system MTF of conventional imaging systems. Furthermore, emerging methods in 3D inverse filtering often improve the fidelity of the topography reproduction even in the presence of aberrations, focus errors and wavelength-dependent spatial frequency response.

Consequently, although there is still significant room to improve, we can safely say that interferometry does work, and it works quite well.

\section{ACKNOWLEDGEMENTS}

The Authors gratefully acknowledge the contributions and useful discussions with Jeremy Coupland (Loughborough University), John Sheridan (University College Dublin), Matthew Thomas (University of Nottingham), Shiguang Wang, Les Deck, Thomas Dresel, and Bruce Truax (Zygo Corporation).

\section{REFERENCES}

[1] Deck, L. L., and Soobitsky, J. A., "Phase-shifting via wavelength tuning in very large aperture interferometers," 3782, 432-442 (1999).

[2] de Groot, P., "Principles of interference microscopy for the measurement of surface topography," Advances in Optics and Photonics 7(1), 1-65 (2015).

[3] de Groot, P., and DiSciacca, J., "Surface-height measurement noise in interference microscopy," Proc. SPIE 10749, 107490Q-1 - 107490Q-9 (2018).

[4] Deck, L. L., "Fourier-transform phase-shifting interferometry," Applied Optics 42(13), 2354-2365 (2003).

[5] Sachs, R., and Stanzel, F. "Interference microscopy for clean air - how optical metrology is improving quality control of fuel injection systems," [Fringe 2013: 7th international workshop on advanced optical imaging and metrology], W. Osten, Ed., Springer Berlin Heidelberg, 96 (2014).

[6] Colonna de Lega, X., and de Groot, P., "Optical topography measurement of patterned wafers," Proc. ULSI Technology CP 788, 432-436 (2005).

[7] Fay, M. F., and Dresel, T., "Applications of model-based transparent surface films analysis using coherencescanning interferometry," Optical Engineering 56(11), 6 (2017).

[8] Deck, L. L., "Model-based phase shifting interferometry," Applied Optics 53(21), 4628-4636 (2014).

[9] Sykora, D. M., and Holmes, M. L., "Dynamic measurements using a fizeau interferometer," Proc. SPIE 8082, 80821R-1 - 80821R-10 (2011).

[10] DiSciacca, J., Gomez, C., Thompson, A., Lawes, S. D. A., Leach, R. K., Colonna de Lega, X., and de Groot, P., "True-color $3 \mathrm{~d}$ surface metrology for additive manufacturing using interference microscopy," Proc. EUSPEN, 145 - 148 (2017).

[11] Gomez, C., Su, R., Thompson, A., DiSciacca, J., Lawes, S., and Leach, R. K., "Optimization of surface measurement for metal additive manufacturing using coherence scanning interferometry," Optical Engineering 56(11), 8 (2017).

[12] Colonna de Lega, X., Dresel, T., Liesener, J., Fay, M., Gilfoy, N., Delldonna, K., and de Groot, P., "Optical form and relational metrology of aspheric micro optics," Proc. ASPE 67, 20-23 (2017). 
[13] Leach, R. K., de Groot, P. J., and Haitjema, H., "Infidelity and the calibration of surface topography measuring instruments," Proc. ASPE (2018).

[14] Goodman, J. W., [Introduction to fourier optics], W. H. Freeman Macmillan Learning, New York (2017).

[15] de Groot, P., Stoner, R., and Colonna de Lega, X., "Profiling complex surface structures using scanning interferometry," US Patent 7,106,454, (2006).

[16] de Groot, P., and Colonna de Lega, X., "Interpreting interferometric height measurements using the instrument transfer function," Proc. FRINGE, 30-37 (2006).

[17] Colonna de Lega, X., and de Groot, P., "Lateral resolution and instrument transfer function as criteria for selecting surface metrology instruments," OSA Proc. Optical Fabrication and Testing, OTu1D (2012).

[18] de Groot, P. J., "A review of selected topics in interferometric optical metrology," Reports on Progress in Physics 82(5), 056101 (2019).

[19] ISO, "9334: Optics and photonics -- optical transfer function -- definitions and mathematical relationships," International Organization for Standardization, Geneva (2012).

[20] Boreman, G. D., [Modulation transfer function in optical and electro-optical systems], SPIE Press, Bellingham (2001).

[21] ISO, "9335: Optics and photonics - optical transfer function - principles and procedures of measurement," International Organization for Standardization, Geneva (2012).

[22] ISO, "Dis 25178-600:201: Geometrical product specifications (gps) - surface texture: Areal — part 600: Metrological characteristics for areal-topography measuring methods (27 february, 2018)," International Organization for Standardization, Geneva (2018).

[23] Novak, E., Ai, C., and Wyant, J. C., "Transfer function characterization of laser fizeau interferometer for highspatial-frequency phase measurements," Proc. SPIE 3134, 114-121 (1997).

[24] Doerband, B., and Hetzler, J., "Characterizing lateral resolution of interferometers: The height transfer function (htf)," Proc. SPIE 5878, 587806-1 - 587806-12 (2005).

[25] Takacs, P. Z., Li, M. X., Furenlid, K., and Church, E. L., "Step-height standard for surface-profiler calibration," Proc. SPIE 1995, 235-244 (1993).

[26] Glaschke, T., Deck, L., and de Groot, P., "Characterizing the resolving power of laser fizeau interferometers," Proc. SPIE 10829, 1082905-1 - 1082905-6 (2018).

[27] de Groot, P., Colonna de Lega, X., Sykora, D. M., and Deck, L., "The meaning and measure of lateral resolution for surface profiling interferometers," Optics and Photonics News 23(4), 10-13 (2012).

[28] Xu, L., Zhou, Y., Zhu, R., Bai, Y., and Liu, S., "New fabrication method of step plate for measuring instrument transfer function of interferometer," Proc. SPIE 11068, 110682U-1 - 110682U-7 (2019).

[29] Deck, L. L., "Method and apparatus for optimizing the optical performance of interferometers," US Patent Application 15/383,019, (2016).

[30] Wang, S., "Coherent phase transfer function degradation due to wave aberrations of a laser fizeau interferometer," Optical Engineering 56(1), 111711-1 - 111711-9 (2017).

[31] Wang, S., "Wave aberration tolerance for the optical design of a laser fizeau interferometer," OSA Technical Digest (online), ITu1A.1 (2017).

[32] Deck, L. L., "Method and apparatus for optimizing the optical performance of interferometers," US Patent Application 20170191821, (2017).

[33] Colonna de Lega, X., "Aberration characterization using frequency domain analysis of low-coherence interferograms," Proc. SPIE 5531, 208-219 (2004).

[34] Lehmann, P., Tereschenko, S., Allendorf, B., Hagemeier, S., and Hüser, L., "Spectral composition of lowcoherence interferograms at high numerical apertures," Journal of the European Optical Society-Rapid Publications 15(1), 1-9 (2019).

[35] Harasaki, A., and Wyant, J. C., "Fringe modulation skewing effect in white-light vertical scanning interferometry," Applied Optics 39(13), 2101 (2000).

[36] Lehmann, P., Tereschenko, S., and Xie, W., "Fundamental aspects of resolution and precision in vertical scanning white-light interferometry," Surface Topography: Metrology and Properties 4(2), 024004 (2016).

[37] Xie, W., Lehmann, P., Niehues, J., and Tereschenko, S., "Signal modeling in low coherence interference microscopy on example of rectangular grating," Optics Express 24(13), 14283-14300 (2016).

[38] de Groot, P., Colonna de Lega, X., Kramer, J., and Turzhitsky, M., "Determination of fringe order in white-light interference microscopy," Applied Optics 41(22), 4571-4578 (2002).

[39] de Groot, P., "Topographical profiling with coherence scanning interferometry," US Patent 8,780,334, (2014). 
[40] Tapilouw, A. M., Chang, Y.-W., Yu, L.-Y., and Wang, H.-W., "Reduction of batwing effect in white light interferometry for measurement of patterned sapphire substrates (pss) wafer," 9960, 996006-996006-8 (2016).

[41] Badami, V. G., Liesener, J., Evans, C. J., and de Groot, P., "Evaluation of the measurement performance of a coherence scanning microscope using roughness specimens," Proc. ASPE, 23-26 (2011).

[42] Totzeck, M., "Numerical simulation of high-na quantitative polarization microscopy and corresponding nearfields," Optik - International Journal for Light and Electron Optics 112(9), 399-406 (2001).

[43] de Groot, P., and Colonna de Lega, X., "Signal modeling for low-coherence height-scanning interference microscopy," Applied Optics 43(25), 4821 (2004).

[44] de Groot, P., Colonna de Lega, X., Liesener, J., and Darwin, M., "Metrology of optically-unresolved features using interferometric surface profiling and rcwa modeling," Optics Express 16(6), 3970 (2008).

[45] Leach, R. K., Weckenmann, A., Coupland, J., and Hartmann, W., "Interpreting the probe-surface interaction of surface measuring instruments, or what is a surface?," Surface Topography: Metrology and Properties 2(3), 035001 (2014).

[46] Su, R., Thomas, M., Leach, R. K., and Coupland, J., "Effects of defocus on the transfer function of coherence scanning interferometry," Optics Letters 43(1), 82-85 (2018).

[47] Coupland, J., Mandal, R., Palodhi, K., and Leach, R., "Coherence scanning interferometry: Linear theory of surface measurement," Applied Optics 52(16), 3662-3670 (2013).

[48] Su, R., Thomas, M., de Groot, P. J., Coupland, J. M., and Leach, R. K., "High fidelity surface measurement through modelling of the surface transfer function in interference microscopy " Proc. SPIE 11102, Paper 111024 (2019).

[49] Frieden, B. R., "Optical transfer of the three-dimensional object," Journal of the Optical Society of America 57(1), 56-66 (1967).

[50] Sheppard, C. J. R., and Cogswell, C. J., “Three-dimensional image formation in confocal microscopy,” Journal of Microscopy 159(2), 179-194 (1990).

[51] Coupland, J. M., and Lobera, J., "Holography, tomography and 3d microscopy as linear filtering operations," Measurement Science and Technology 19(7), 074012-1 - 074012-12 (2008).

[52] Su, R., Wang, Y., Coupland, J., and Leach, R., "On tilt and curvature dependent errors and the calibration of coherence scanning interferometry," Optics Express 25(4), 3297-3310 (2017). 